\title{
Pituitary adenoma and orbital haemangioma: a case report
}

\author{
R. J. GALVIN AND B. E. KENDALL \\ From the Maida Vale Hospital, London, and the National Hospitals for Nervous Diseases, Queen Square, \\ London
}

SUMMARY A 43-year-old woman with a pituitary tumour had unexplained right proptosis. Orbital $x$-rays and ultrasound were normal, but computed tomography showed an intraconal tumour, which had typical features of a cavernous haemangioma on carotid angiography. Both tumours were resected and confirmed histologically.

\begin{abstract}
About $8 \%$ of most series of intracranial tumours are pituitary adenomata (Zülch, 1965), the majority of these being chromophobe. The most usual presentation is with bitemporal field defects and visual failure (Martins, 1974). Cavernous haemangiomata are the commonest type of orbital tumour (Reese, 1964). Their cardinal symptom is proptosis, but they may also produce papilloedema, optic atrophy, and diplopia (Duke-Elder, 1974). We report here the rare, and presumably chance, association of both these tumours in the same patient. Although clinically a difficult problem, the patient displayed classical radiological features of both these tumours, The value of computed tomography is stressed in this situation.
\end{abstract}

\section{Case report}

The patient, a 43-year-old woman, had been noted by her friends to have right proptosis 1 year before being referred to hospital. This had slowly progressed. Three months later she had developed slight intermittent diplopia, mainly on upgaze, with vertical displacement of the images. For 6 months she noticed a faint 'haziness' of vision bilaterally, more marked in her right eye.

She had had decreased libido and secondary amenorrhoea for 26 years after a normal menarche, and for many years she had tended to gain weight and suffered from cold intolerance. She had been unsuccessfully treated with 'thyroid tablets' 10 years ago. For several years she had worn a wig because of sparse capital hair.

She was initially assessed ophthalmologically and was referred to the Maida Vale Hospital after a

Address for reprints: Dr R. J. Galvin, National Hospitals for Nervous Diseases, Queen Square, London WC1N 3BG
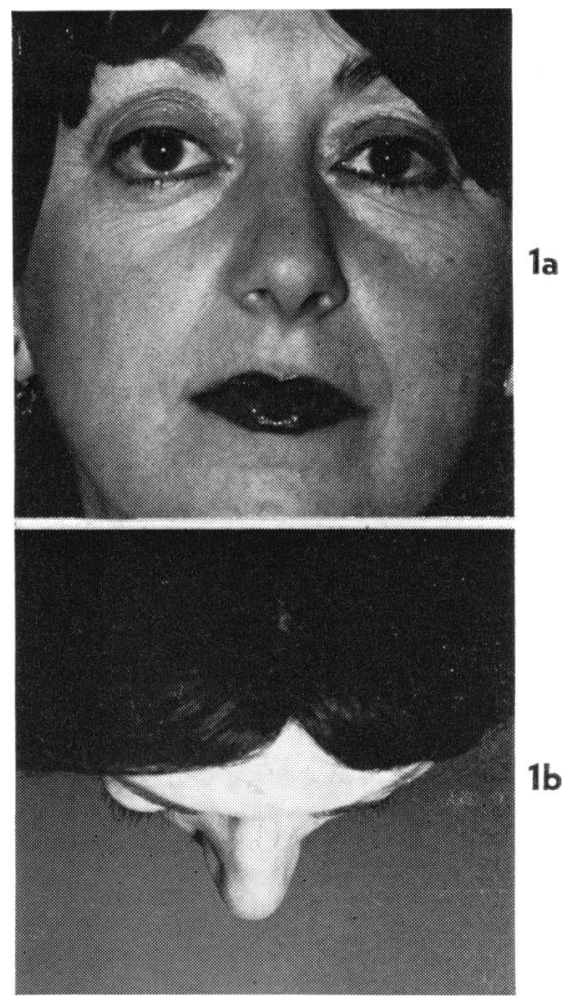

Figs. 1a, b Right proptosis viewed from in front and above

normal orbital ultrasound study and with a skull $x$-ray showing an enlarged pituitary fossa.

Examination showed a moderately overweight, normotensive woman. Scalp hair was thin and dry, and pubic and axillary hair, though sparse, was of normal distribution. There was $5 \mathrm{~mm}$ of axial proptosis on the right (Fig. 1). Elevation of the right 


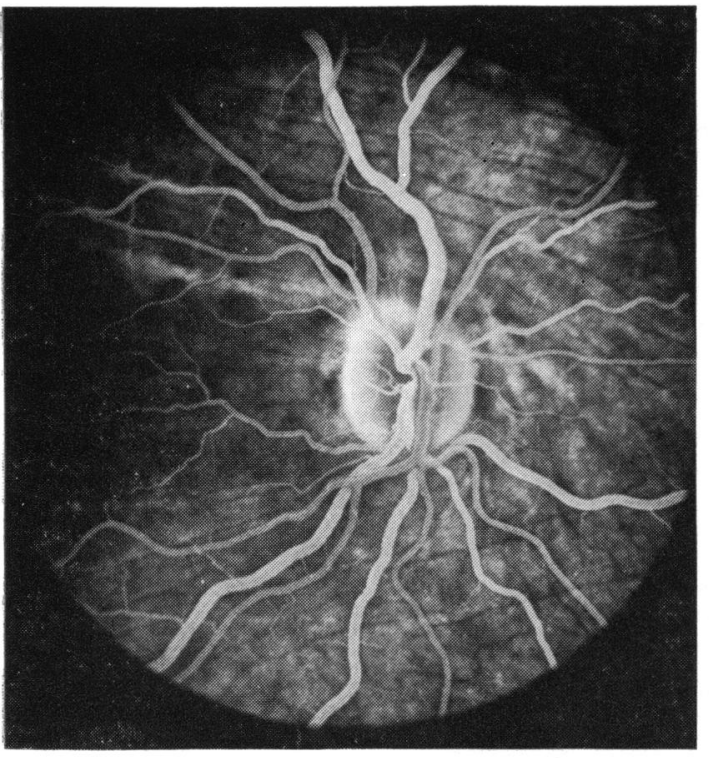

$2 a$

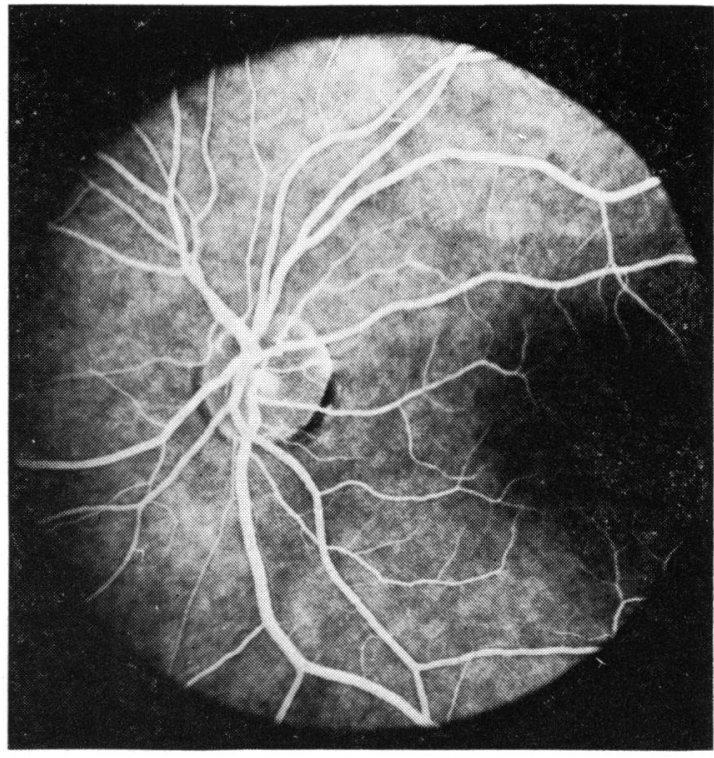

$2 b$

Figs. 2a, b Fluorescein angiogram. Both sides are given for comparison. There is slight disc swelling on the right and choroidal folds are visible

eye was restricted to $50 \%$, and this was more marked on adduction. There were no orbital bruits. Visual acuity corrected to $6 / 7 \cdot 5$ right and $6 / 6$ left. There was hereditary deuteranomaly. Initial Goldmann and tangent screen fields were full, but repeat testing after 6 weeks revealed a subtle right caecocentral defect and left upper temporal restriction. The right disc was slightly hyperaemic; there were choroidal folds superotemporally on the right.

Investigations. The erythrocyte sedimentation rate was raised at $37 \mathrm{~mm}$ in 1 hour. The remaining haematological and biochemical indices, including plasma and urinary osmolalities, were normal. Endocrinological studies showed a euthyroid and eucortisol state, and an insulin tolerance test was normal. There was marked elevation of serum prolactin to greater than $4900 \mathrm{mU} / 1$ (normal 0 to $360 \mathrm{mU} / \mathrm{l})$. Fluorescein angiography confirmed the right disc oedema and choroidal folds (Fig. 2). Pattern visual evoked responses were bilaterally abnormal in wave form, more so on the right, and half field responses were consistent with early compression involving the decussating fibres at the chiasm.

Radiological investigations. Plain $x$-rays and tomograms of the skull showed asymmetrical ballooning of the sella turcica typical of a pituitary tumour (Fig. 3). There was no calcification or other ab- normality. In particular the orbits appeared normal on polytomography. Orbital B-scan ultrasound was normal. Computed tomography (Fig. 4) showed a mass of increased attenuation, without calcification, which enhanced homogeneously, filling the ballooned sella and suprasellar cisterns. There was an evenly dense, rounded, intraconal mass with clear-cut edges displacing the right globe and obviously causing the proptosis. In common with most orbital masses it enhanced considerably after intravenous contrast medium.

Right carotid angiography showed a diffuse capillary blush supplied by hypophyseal arteries outlining the pituitary and suprasellar tumour. There was no displacement of any vessels by this tumour. The superior ophthalmic vein was deviated and curved superiorly and was somewhat narrowed but not obstructed by the intraconal mass. Lakes of contrast filled within part of the tumour in the venous phase (Fig. 5).

Pneumoencephalography confirmed the outline of the suprasellar mass and showed the anterior recesses of the third ventricle to be elevated and splayed apart by it.

Operation. A right frontal craniotomy was performed for excision of the pituitary tumour (Mr L. Symon). The tumour proved difficult to detach from the undersurface of the chiasm, but it was excised fairly completely with its capsule. The 


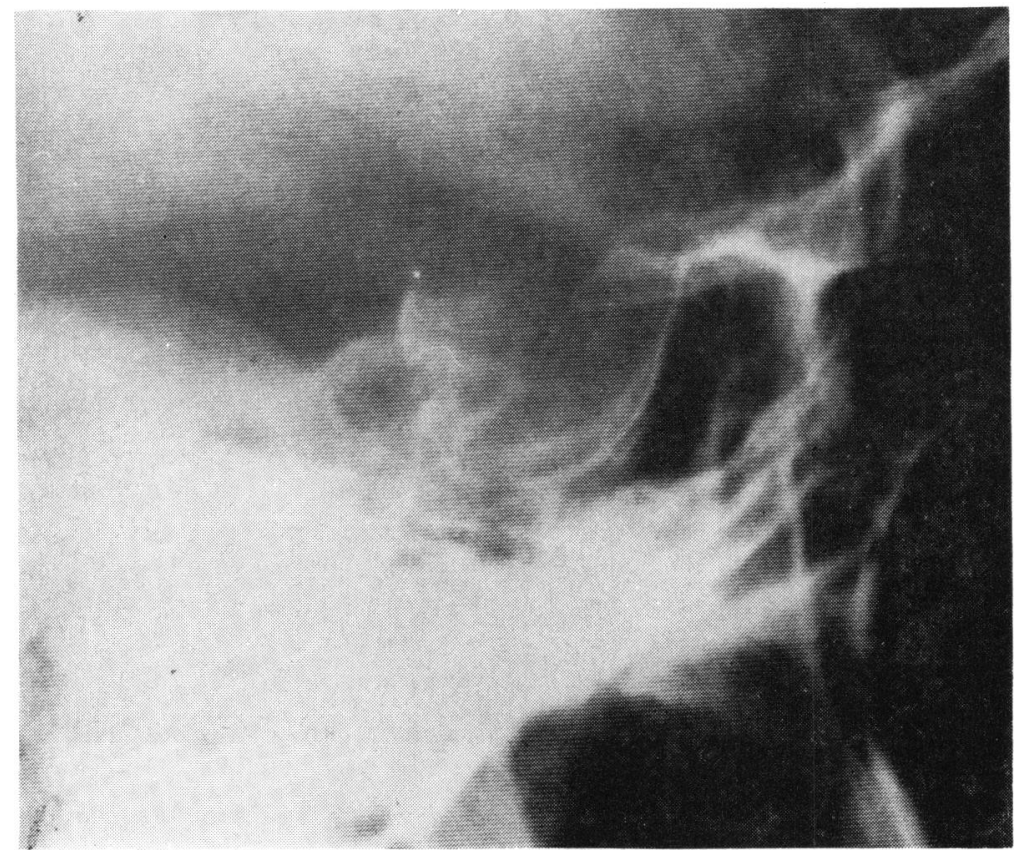

Fig. 3 Skull x-ray. Lateral projection. The pituitary fossa is asymmetrically ballooned by an intrasellar mass

craniotomy was extended into the orbit, and a black rounded mass $16 \times 10 \mathrm{~mm}$ was found in the lateral part of the orbit, running from behind the globe almost to the apex of the orbit. It was easily removed in its entirety. Postoperatively the patient had a dense bitemporal hemianopia but with macular sparing. She otherwise made an uneventful recovery.

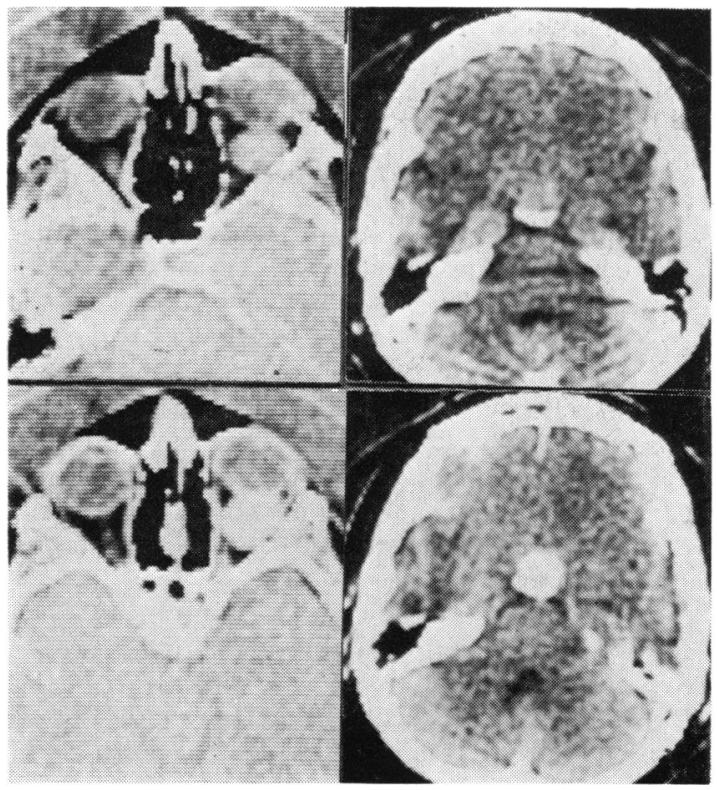

Histology. The tumours showed classical histological features of chromophobe adenoma and cavernous haemangioma.

\section{Discussion}

The ballooned sella turcica and the capillary blush supplied by hypophyseal arteries to a tumour extending from the sella through the suprasellar cisterns are the classical radiological features of a pituitary adenoma. The computed tomographic features are also those commonly found in pituitary adenomas, $75 \%$ of which show increased attenuation on computed tomography and most of which show homogeneous enhancement after intravenous contrast medium. Pneumoencephalography, which is performed routinely in our unit

Fig. 4 Computed tomography before (above) and after intravenous contrast medium (below). The scans were taken at the level of the orbit and sella (window level $=-6$, window width $=100$ ) and suprasella (window level +18 , width 40). There is a spherical intraconal mass with clear-cut edges in the right orbit displacing the globe anteriorly. It is of high attenuation and homogeneous but without evidence of calcification. There is another mass of greater-than-brain attenuation without calcification in the pituitary fossa extending into the suprasellar cistern. The right side of the dorsum is deviated posteriorly. Both tumours show homogeneous enhancement after contrast medium 
Fig. 5 Right internal carotid angiotomogram. Venous phase. There is a cluster of dilated veins (see arrows) in the middle and posterior thirds of the right orbit within the mass. These appearances are typical of a cavernous haemangioma

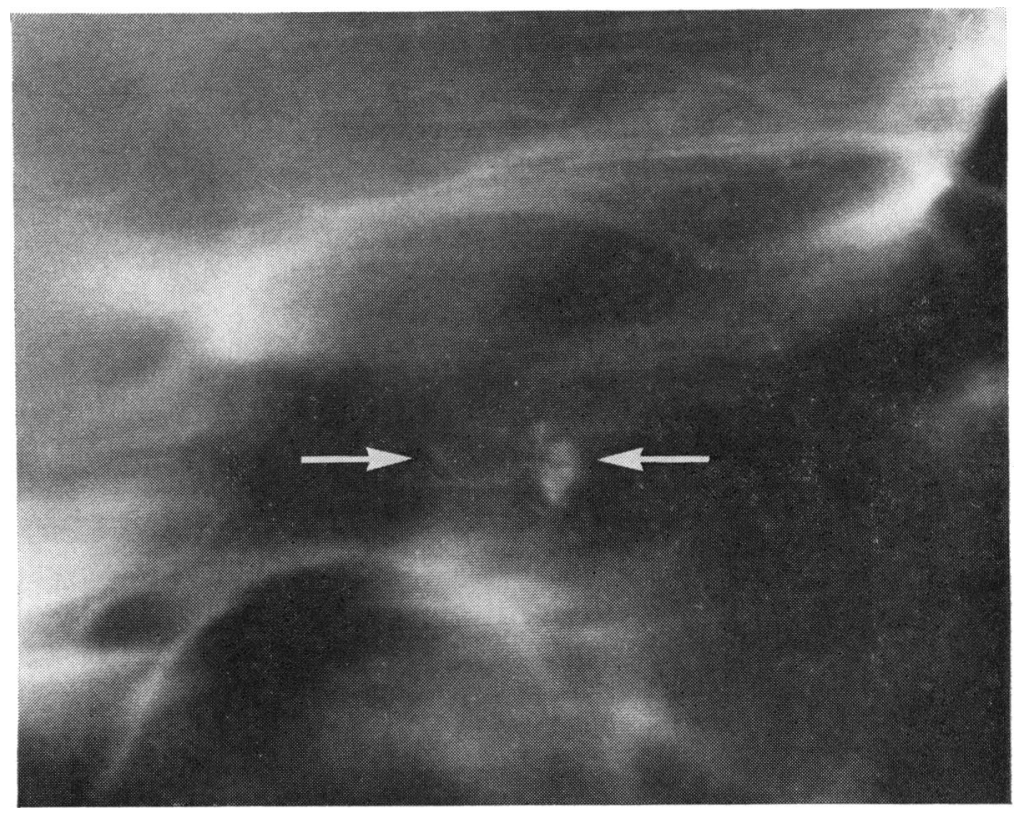

to show the relationship of a pituitary mass to optic chiasm and nerves prior to surgery, further confirmed that the tumour extended from the pituitary fossa into contact with the optic chiasm.

In the presence of a pituitary tumour and with a normal orbital ultrasound the possibility of an endocrine exophthalmos was considered clinically. It seemed unlikely with choroidal folds, pronounced unilateral involvement, and absence of thyrotrophic abnormalities. Computed tomography left no doubt that the proptosis was due to an intraconal orbital tumour and showed no evidence of thickening of the rectus muscles such as occurs in thyrotrophic exophthalmos. Computed tomography shows the localisation and extent of intraorbital tumours in the great majority of cases (Lloyd and Ambrose, 1977). It is usually possible to distinguish benign intraconal tumours, which typically show clearly demarcated edges from infiltrating orbital masses, which are much less well defined and may obliterate the outlines of normal structures within the orbit. A haemangioma is the commonest cause of a rounded, well-demarcated, homogeneous, high-attenuation mass, though it is precisely similar to the lesscommon neurofibroma and to some optic sheath meningiomas. The latter, however, may contain calcification. There is generally no difficulty in distinguishing an optic nerve glioma, which tends to be orientated in the axis of the nerve and inseparable from it. Since there is no blood-brain barrier in the orbit, all intraorbital tumours may enhance and cannot be distinguished by using this technique.
Most orbital haemangiomas show abnormal circulation. This is sometimes rapid, with arteriovenous shunting, but the appearances shown in our patient of cavernous blood spaces outlining with contrast medium in the venous phase are typical of this type of lesion. There is, in addition, a variable amount of solid tumour which causes a mass effect. The deviation of the ophthalmic veins without obstruction is helpful in distinguishing localised benign masses from infiltrating orbital lesions.

We are grateful to Professors W. I. McDonald and L. Symon, who kindly permitted us to publish this case. Mr M. D, Sanders permitted us to publish Figs. 1 and 2. Photographic. assistance was provided by the Departments of Medical Illustration at the National Hospital, Queen Square, and the Middlesex Hospital.

\section{References}

Duke-Elder, S. (1974). The ocular adnexa. In System of Ophthalmology, Vol. 10, Part II, pp. 1086-1096. Kimpton: London.

Lloyd, G. R. S., and Ambrose, J. A. E. (1977). An evaluation of computerised axial tomography in the diagnosis of orbital space occupying lesions. Computerized Axial Tomography in Clinical Practice, pp. 154-160. Edited by G. H. du Boulay and I. F. Moseley. Springer: New York.

Martins, A. N. (1974). Pituitary tumours and suprasellar cysts. Handbook of Clinical Neurology, Vol. 17, pp. 375440. Edited by P. J. Vinken and E. W. Bruyn. Elsevier: New York.

Reese, A. B. (1964). Tumours of the Eye, 2nd edn., p. 532. Hoebner: New York.

Zülch, K. J. (1965). Brain Tumours: Their Biology and Pathology, 2nd Amer. edn., pp. 78-237. Springer: New York. 